\title{
Using Optimized Collision Energies and High Resolution, High Accuracy Fragment Ion Selection to Improve Glycopeptide Detection by Precursor Ion Scanning
}

\author{
Judith Jebanathirajah, ${ }^{*}$ Hanno Steen, ${ }^{*}$ and Peter Roepstorff \\ Department of Biochemistry and Molecular Biology, University of Southern Denmark, Odense, Denmark
}

\begin{abstract}
Glycosylation is the most widespread protein modification and is known to modulate signal transduction and several biologically important interactions. In order to understand and evaluate the biological role of glycosylation it is important to identify the glycosylated protein and localize the site glycosylation under particular biological conditions. To identify glycosylated peptides from simple mixtures, i.e., in-gel digests from single SDS PAGE bands we performed high resolution, high accuracy precursor ion scanning using a quadrupole TOF instrument equipped with the $Q_{2}$ pulsing function. The high resolving power of the quadrupole TOF instrument results in the selective detection of glycan specific fragment ions minimizing the interference of peptide derived fragment ions with the same nominal mass. Precursor ion scanning has been previously described for these glycan derived ions. However the use of this method has been limited by the low specificity of the method. The analysis using precursor ion scanning can be applied to any peptide mixture from a protein digest without having previous knowledge of the glycosylation of the protein. In addition to the low femtomole (nanomolar) detection limits, this method has the advantage that no prior derivatization or enzymatic treatment of the peptide mixtures is required. (J Am Soc Mass Spectrom 2003, 14, 777-784) (C) 2003 American Society for Mass Spectrometry
\end{abstract}

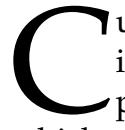
urrent genome sequencing efforts have resulted in a remarkable resource of protein and putative protein sequences. These protein sequences, which are stored in accessible databases, increase the facility with which proteins may be identified using mass spectrometry. As protein identification at the subpicomole level becomes routine, there is a shift in interest from simple protein identification, i.e., correlation of a sample spot on a PAGE gel with an accession number, to protein characterization with special emphasis on the analysis of the co- and posttranslational protein modifications. One of these modifications is protein glycosylation, which is the most common protein modification. It is estimated that $50 \%$ of all proteins are glycosylated [1]. This modification plays a major structural role but is also heavily involved in cell-cell recognition and modulating molecular interaction. Furthermore, there is the increasing notion that reversible glycosylation plays a pivotal role in signaling mechanisms [2]. In order to understand and evaluate the biological role of glycosylation in detail it is important

Published online June 2, 2003

Address reprint requests to Dr. P. Roepstorff, Department of Biochemistry and Molecular Biology, University of Southern Denmark, Campusvej 55, DK-5230 Odense, Denmark. E-mail: roe@bmb.sdu.dk

${ }^{*}$ Current address: Department of Cell Biology, Harvard Medical School, 240 Longwood Ave., Boston, MA 02115, USA. to analyze the glycosylation. A comprehensive analysis comprises three steps, each of them is a challenge on its own: (1) The identification of glycosylated proteins and peptides, (2) the localization of the glycosylation sites, and (3) the elucidation of the glycan structure. One of the major problems with glycosylation analysis is the fact that this modification is normally highly heterogeneous such that it induces a fairly undefined mass shift that can easily exceed the mass of the peptide. In contrast, "simple" modifications such as phosphorylation or acetylation induce well-defined mass shift (or multiples thereof).

One approach that can be used to identify glycosylated peptides is precursor ion scanning for glycan derived fragment ions. As further MS/MS studies of the identified glycopeptides can be performed immediately after their identification, much information about the glycopeptide may be derived. Subsequent mass spectrometric experiments elucidating the sequence or structure of the peptide and the glycan can be executed, in order to localize the glycosylation site. Information with respect to the compositions and the sequences of the glycans can also be obtained. The characteristic fragment ions used for this purpose are normally the "reporter" oxonium ions of hexose at $m / z$ 163.060, of N-acetylhexosamines at $\mathrm{m} / \mathrm{z} 204.084$, and of hexoylhexosamine at $m / z 366.139$ [3], but much larger oxonium 
ions have also recently been used [4]. These fragment ions enable the selective detection of glycosylated peptides using precursor ion scanning or skimmer fragmentation routines on triple or single quadrupole mass spectrometers, respectively. However, Carr and coworkers reported a lack of specificity for these precursor ion experiments whereby other nonglycosylated peptides produce signals in the precursor ion scan as the produce other peptide-derived fragment ions that have the same nominal mass as the characteristic reporter ion [5]. This problem is commonly encountered when low resolution triple quadrupole mass spectrometers are used for precursor ion experiments. In order to successfully use a triple quadrupole mass spectrometer for precursor ion scanning experiments characteristic fragment ions have to be unique within $\pm 0.5 \mathrm{Da}$. This factor has prevented the widespread use of precursor ion experiments for protein modification analysis in general and in particular for protein glycosylation analysis, as many characteristic fragment ions are not unique within the required limits. However, in some cases the selectivity of precursor ion experiments can be significantly increased by using high resolution, high accuracy quadrupole TOF mass spectrometers equipped with so-called $Q_{2}$ pulsing, which enables precursor ion experiments on quadrupole TOF instruments with similar sensitivities as on triple quadrupole mass spectrometers. Using this type of tandem mass spectrometer for precursor ion scanning it is sufficient if the reporter fragment ion is unique within $\pm 0.03 \mathrm{Da}$ as compared with the $\pm 0.5 \mathrm{Da}$, required when using the traditional triple quadrupole mass spectrometer. Since the second mass analyzer in quadrupole TOF instrument, the TOF, cannot be used as filter, the acquired spectra are not true precursor ion spectra but reconstructed spectra. This implies that longer scan times are necessary, thereby limiting this approach. The application of this high resolution precursor ion scanning was recently demonstrated by Steen et al. for the selective detection of various protein modifications such as tyrosine phosphorylation and nitration, tryptophan bromination, and proline hydroxylation by utilizing the corresponding immonium ions [6-9]. All the immonium ions used for high resolution, high accuracy precursor ion scanning showed a mass difference less than $0.1 \mathrm{Da}$ with respect to the interfering peptidederived fragment ions with the same nominal masses. This mass shift was adequately large such that the resolution of the reporter ions from the interfering fragment ions was feasible. The characteristic mass difference observed for the above-described modifications is the result of a higher incidence of mass deficient elements, such as phosphorus, oxygen, or bromine. Glycan derived-oxonium ions are not strictly speaking mass deficient, nevertheless they show resolvable mass difference with respect to other interfering fragment ions when using high resolution, high accuracy fragment ion selection which would increase the specificity of precursor ion scanning for the selective identification of glycopeptides within complex peptide mixtures. Here we investigate the use of the high resolution precursor ion scans for the identification of previously unknown glycopeptides in complex peptide mixtures. We first tested several synthetic glycopeptides to establish and optimize conditions prior to applying the high resolution scan to "real" samples. We then show the applicability of these methods in locating previously unknown glycopeptides in complex protein digests.

\section{Experimental}

\section{Chemicals and Compounds}

Chemicals and the standard glycoproteins ovalbumin and lactoglobulin were obtained from Sigma-Aldrich (St. Louis, MO). High purity solvents were purchased from Labscan (Dublin, Ireland). The synthetic glycopeptides GFYFNKPSGYGSSSRRA, PTTTPIST, ITTTTTVT, and VITAFNEGLK (glycosylation sites are in bold face) were obtained from the Carlsberg Research Center (Copenhagen, Denmark). Sequencing grade trypsin was purchased from Roche Diagnostics (Mannheim, Germany).

\section{Protein Expression and Purification}

Urokinase plasminogen activator receptor (UPAR), expressed in Chinese hamster ovary cells and in SN194 Snyder cells. Proteins were separated by SDS-PAGE (4-12\% NuPage; Novex, San Diego, CA) and visualized by colloidal Coomassie blue staining (Colloidal Blue Staining kit, Novex). The appropriate gel band of interest were excised and subjected to in-gel reduction, alkylation and tryptic digestion as described previously [10].

\section{Peptide Purification}

All digests were desalted and concentrated on a microcolumn of POROS R2 material (Applied Biosystems, Framingham, MA) packed into GELoader tips (Eppendorf, Hamburg, Germany) as described previously [11, 12]. Columns were eluted using $60 \%$ methanol in $5 \%$ formic acid directly into nanoelectrospray needles (Proxeon Biosystems A/S, Odense, Denmark) and the eluted fraction was subjected to mass spectrometric analysis.

\section{Mass Spectrometry}

Precursor ion experiments were performed on a QSTAR Pulsar quadrupole time of flight tandem mass spectrometer (AB/MDS-Sciex, Toronto, Canada) equipped with a nanoelectrospray ion source (Proxeon Biosystems). Precursor ion scanning experiments were acquired with the $Q_{2}$ pulsing function turned on using a dwell time of $50 \mathrm{~ms}$ at a step size of $1 \mathrm{Da}$. 


\section{Results and Discussion}

\section{Interfering Peptide Fragment Ions}

The use of the various sugar oxonium ions $\left(\mathrm{Hex}^{+}\right.$at $\mathrm{m} / \mathrm{z}$ 163.060, $\mathrm{HexNAc}^{+}$at $m / z 204.084$ and HexHexNAc${ }^{+}$at $\mathrm{m} / \mathrm{z}$ 366.139) were investigated for the detection of glycosylated peptides using precursor ion scanning with high resolution, high accuracy fragment ion selection on a quadrupole TOF mass spectrometer. These glycan specific reporter ions and their nominal masses were first described and used by Carr et al. for the selective detection of glycopeptides [3,5]. However, a lack of selectivity was reported resulting from numerous peptide-related fragment ions with the same nominal mass that cannot be resolved when low resolution triple quadrupole tandem mass spectrometers are used. These potentially interfering peptide fragment ions are listed in Table 1. Only a, b, and y ions are considered but not the concomitant loss of water or ammonia.

\section{Optimizing Collision Energies}

First, the abundance of characteristic "reporter"fragment ion as a function of the collision energies was determined. This was performed in an effort to maximize the sensitivity of the experiments as the sensitivity of a given precursor ion experiment is directly proportional the absolute abundance of the reporter fragment ion. The collision energy was ramped over a wide range for several different model glycopeptides with varying $\mathrm{N}$-linked and O-linked glycan structures. Several conclusions could be confirmed/drawn from those measurements:

1. Glycans are extremely labile; very little collision energy is required to induce the cleavage of the glycosidic bonds.

2. O-linked glycans are more labile than N-linked glycans.

3. Oxonium ions derived from O-linked glycans have maximum intensities at collision energies approximately $1 / 30$ of the numerical value of the precursor $\mathrm{m} / \mathrm{z}$ value.

4. Oxonium ions derived from $\mathrm{N}$-linked glycans reach a maximum at collision energies of approximately $1 / 20$ of the numerical value of the precursor $\mathrm{m} / \mathrm{z}$ value (these estimates hold true for QSTAR instruments and have to be optimized for the different instrument types and models).

5. HexHexNAc oxonium ion at $m / z 366$ reaches a maximum at a lower collision energy than the HexNAc oxonium ion at $\mathrm{m} / \mathrm{z} 204$, which might indicate that the latter ion is formed by secondary fragmentation of the former one (these estimates hold true for QSTAR quadrupole TOF instruments and have to be optimized for the different instrument types and models).
Table 1. List of exact masses and amino acid compositions of all possible a-, b-, and y-type fragment ions of unmodified peptides giving rise to signals at nominal masses of 163, 204 and 366

\begin{tabular}{|c|c|}
\hline Fragment Ion & $m / z$ \\
\hline Hex oxonium ion & 163.060 \\
\hline $\mathrm{y}_{2} \mathrm{GS}$ & 163.072 \\
\hline HexNAc oxonium ion & 204.084 \\
\hline $\mathrm{a}_{2} \mathrm{AC}$ & 204.081 \\
\hline$a_{3}$ GSS & 204.098 \\
\hline $\mathrm{y}_{2} \mathrm{AN}$ & 204.098 \\
\hline $\mathrm{y}_{3} \mathrm{AGG}$ & 204.098 \\
\hline $\mathrm{y}_{2} \mathrm{GQ}$ & 204.098 \\
\hline $\mathrm{Y}_{2} \mathrm{GK}$ & 204.135 \\
\hline HexHexNAc oxonium ion & 366.139 \\
\hline$a_{3}$ DDY & 366.130 \\
\hline $\mathrm{a}_{3} \mathrm{MMM}$ & 366.134 \\
\hline$a_{3}$ DFM & 366.149 \\
\hline$a_{3}$ ETY & 366.167 \\
\hline$a_{3} M V Y$ & 366.185 \\
\hline $\mathrm{a}_{3} \mathrm{HQO}$ & 366.189 \\
\hline$a_{3} F F V$ & 366.218 \\
\hline$a_{3} \mathrm{HKO}$ & 366.225 \\
\hline$a_{3}$ HKK & 366.262 \\
\hline$b_{3}$ DSY & 366.130 \\
\hline $\mathrm{b}_{3} \mathrm{AMY}$ & 366.149 \\
\hline $\mathrm{b}_{3} \mathrm{FMS}$ & 366.149 \\
\hline $\mathrm{b}_{3} \mathrm{HNN}$ & 366.153 \\
\hline $\mathrm{b}_{3} \mathrm{HMP}$ & 366.160 \\
\hline $\mathrm{b}_{3}$ TTY & 366.167 \\
\hline $\mathrm{b}_{3} \mathrm{DHL}$ & 366.178 \\
\hline $\mathrm{b}_{3} \mathrm{EHV}$ & 366.178 \\
\hline $\mathrm{b}_{3} \mathrm{DHI}$ & 366.178 \\
\hline $\mathrm{b}_{3} \mathrm{AFF}$ & 366.182 \\
\hline $\mathrm{y}_{3} \mathrm{EMS}$ & 366.133 \\
\hline $\mathrm{y}_{3} \mathrm{DMT}$ & 366.133 \\
\hline $\mathrm{y}_{3} \mathrm{AEF}$ & 366.166 \\
\hline $\mathrm{y}_{3}$ PSY & 366.166 \\
\hline$y_{3} A L Y$ & 366.203 \\
\hline $\mathrm{y}_{3}$ AIY & 366.203 \\
\hline$y_{3}$ FIS & 366.203 \\
\hline$y_{3}$ FTV & 366.203 \\
\hline$y_{3}$ FLS & 366.203 \\
\hline $\mathrm{y}_{3} \mathrm{HIP}$ & 366.214 \\
\hline $\mathrm{y}_{3} \mathrm{HLP}$ & 366.214 \\
\hline
\end{tabular}

In addition to increasing the sensitivity, the selectivity also profits from these optimizations. If the optimum collision energy for the formation of the reporter fragment ion is far below or above the energy used for the fragmentation of the peptide backbone, the formation of potentially interfering fragment ions is considerably reduced. This is observed for the glycopeptides tested whereby the glycan fragments at much lower collision energies than the peptide backbone. These extremely low collision energy settings are distinct from most other types of precursor ion scanning methods used for protein modification analysis that require fairly high collision energies in order to generate the reporter fragment ion of interest. In the case of e.g., nitrotyrosine or phosphotyrosine, collision energy settings that cor- 
respond to one tenth of the $\mathrm{m} / \mathrm{z}$ value proved to be the collision energy setting for the most efficient generation of the immonium ion of interest. The precursors of -79 scanning method is fairly insensitive with respect to increasing the collision energy settings as the $\mathrm{PO}_{3}^{-}$ion does not significantly dissociate further once it is formed.

Although the lability of the glycosidic bond is favorable for the sensitive and selective detection of glycopeptides by precursor ion scanning, it affects the fragmentation of the peptide backbone negatively as the peptide fragmentation often only occurs as a secondary fragmentation process. The peptide backbone fragmentation commonly shows lower yields than primary fragmentation process, i.e., the glycan fragmentation. In order to induce peptide backbone cleavage to derive peptide sequence information fairly harsh CID conditions are required. This characteristic of heteroconjugates comprising peptides and other entities with glycosidic bonds, such as glycopeptides or nucleic acid peptide crosslinks, often makes it difficult to infer sequence information from MS/MS data [13, 14].

\section{Precursors of $m / z 163$}

A synthetic glycopeptide with the sequence GFYFNKPSGYGSSSRRA was used for this experiment. The peptide was glycosylated at Ser-8 (marked in bold face) and has two mannose residues attached. In order to test the specificity of the precursors of $(\mathrm{m} / \mathrm{z} 163)$ experiment, an excess of synthetic glycopeptide was spiked into an myoglobin/lactoglobulin digest such that the ion signal of the glycopeptide was observable in the mass spectrum of the peptide mixture (see Figure 1a; marked with an asterisk). Approximately 2 picomoles were added to 1 picomole of digest. The low-resolution precursors of $\mathrm{m} / \mathrm{z}(163 \pm 0.25)$ experiment of this peptide mixture is shown in Figure 1b. Even though the precursor ion spectrum was acquired at low collision energy settings optimized for selective glycopeptide detection, i.e., maximum oxonium ion abundance and minimum peptide backbone fragmentation, a significant number of non-specific ion signals are observable apart from the major ion signal at $\mathrm{m} / \mathrm{z} 551.8$ that is attributable to the excess of added glycopeptide. This underscores the notion that this type of low-resolution precursor ion experiment shows a lack in selectivity, i.e., when a low-resolution instrument such as a triple quadrupole mass spectrometer is used for this type of tandem mass spectrometric experiment. The corresponding high resolution, high accuracy precursors of $m / z(163.06 \pm 0.02)$ experiment is shown in Figure 1c. The gain in selectivity resulting from the high resolution, high accuracy fragment ion selection is obvious: The ion signal attributable to the glycopeptide is the major ion signal, but all other nonspecific ion signals are greatly reduced.

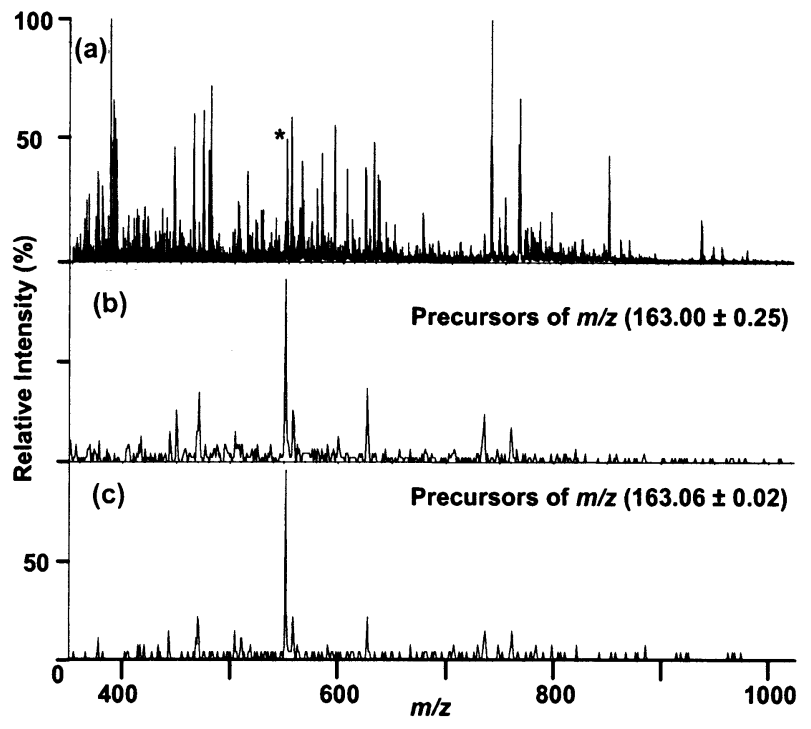

Figure 1. (a) is a mass spectrum of a complex tryptic digest of lactoglobulin and myoglobin with a synthetic, glycosylated peptide spiked into the digest. The synthetic peptide has two hexoses attached via a O-glycosidic linkage. (b) displays the low resolution precursors of $\mathrm{m} / \mathrm{z} 163$ ion scan for the complex mixture shown in (a) whereas the (c) shows the high resolution precursors of $m / z 163$ scan. A clear increase in selectivity is observed in the case of the high resolution scan.

\section{Precursors of $m / z 204$}

A similar set of experiments was performed for the $\mathrm{N}$-acetylhexosamine oxonium ion at $\mathrm{m} / \mathrm{z}$ 204.08. The glycopeptide used for the previous set of experiments could not be used, as it did not had any N-acetylhexosamine modification. Instead, a glycosylated conotoxin (XCCXDGW*CCTAAP ; X $=\gamma$-carboxyglutamic acid, $\mathrm{W}^{*}=$ bromotryptophan, $\mathrm{P}^{\#}=$ hydroxyproline) which had a GalNAc-Gal moiety attached to the threonine residue (marked in bold face) was used [15, 16]. Interestingly, this glycopeptide did not give rise to a hexose oxonium ion at $\mathrm{m} / \mathrm{z} 163$ although it carries a hexose moiety. This observation has been described earlier for other glycopeptides [5]; according to Huddleston et al. the hexose oxonium ion at $m / z 163$ is fairly selective for mannose-rich glycopeptides. This glycosylated conotoxin was spiked into a tryptic digest taken from an ongoing protein identification project. The mass spectrum of the resulting peptide mixture, shown in Figure 2a, demonstrates the complexity of the mixture. The results of the low and the high resolution precursor ion experiments for the selective detection of the precursors of $\mathrm{m} / \mathrm{z}(204.00 \pm 0.25)$ and $\mathrm{m} / \mathrm{z}(204.08 \pm$ $0.02)$ respectively are shown in Figure $2 b$ and $c$. The precursor ion experiment was acquired ramping the collision energy from 20 to $55 \mathrm{~V}$ for the $\mathrm{m} / \mathrm{z}$ range 400 to 1100 . Both precursor ion spectra look very similar with respect to signal to noise ratio as well as total ion count (the count rate is reduced by less than $20 \%$ in the case of the high resolution setting): Both spectra display the doubly charged glycosylated conotoxin at $m / z$ 966.7, its 


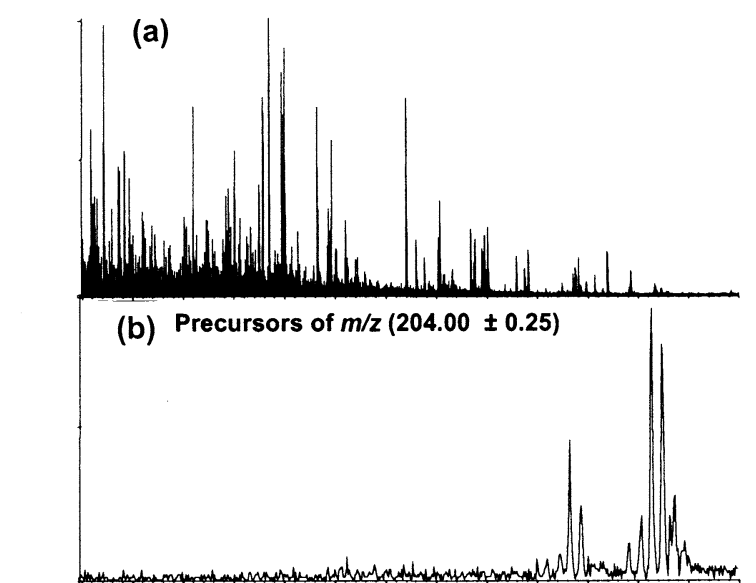

(c) Precursors of $\mathrm{m} / \mathrm{z}(204.08 \pm 0.02)$

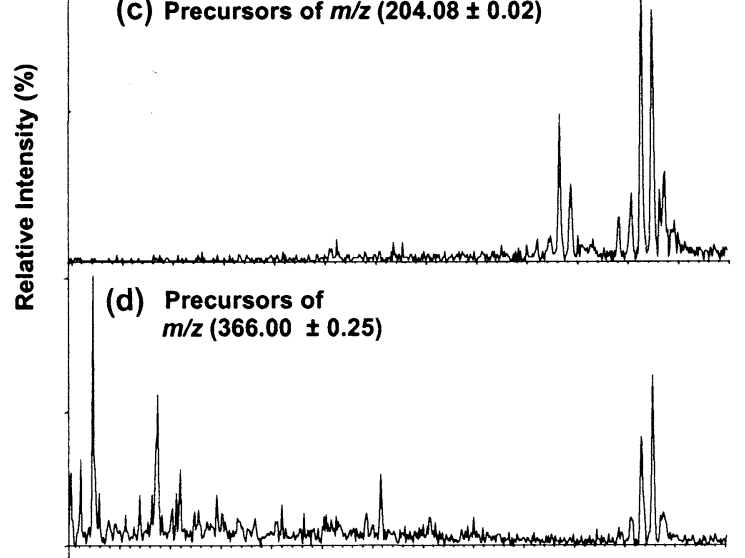

(e) Precursors of $m / z(366.14 \pm 0.02)$

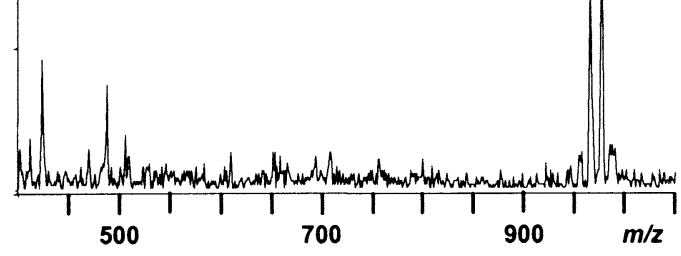

Figure 2. (a) is a MS1 spectrum of a tryptic digest spiked with a glycosylated conotoxin. (b) is the low resolution precursors of $\mathrm{m} / \mathrm{z}$ 204 scan, and (c) is the high resolution precursor ion scan. Both precursor ion scans were performed at low collision energy conditions optimized to reduce nonspecific signals and the high resolution scan does not offer greater specificity at these particular conditions. (d) and (e) display low and high resolution precursors of $m / z 366$ ion scans respectively. A significant increase in selectivity is shown by the high resolution precursor of $\mathrm{m} / \mathrm{z} 366$ scan compared with the low resolution scan.

mono sodium adduct, and its counterparts without the Gal-residue. At this low collision energy setting the precursors of $m / z 204$ is remarkably specific also in the low resolution mode. Although more interfering isobaric peptide fragment ions are expected for $m / z 204$ compared to $m / z 163$ (see Table 1 ). The observation that the precursors of $m / z 204$ are very specific at low collision energies was confirmed by other experiments with standard glycoprotein digests as well as digests from real samples (data not shown). A similar precursor ion experiment was performed using collision energies ramped from 40 to $110 \mathrm{~V}$ over the same $m / z$-range, i.e., at collision energies used for other precursor ion experiments where a characteristic immonium ion is used as reporter ion. Nonspecific ion signals are observable for the high and the low resolution experiment (data not shown). No signals for the glycopeptide were observed any longer indicating that the oxonium ion undergoes further fragmentation under those "higher energy" CID conditions. This stresses the importance of adjusting the collision energy for the given compound class by using standard compounds for preliminary experiments (see above).

\section{Precursors of $m / z 366$}

The same peptide mixture used in the previous experiment was employed for testing the precursor of $m / z 366$ experiments. The collision energies were ramped from 15 to $40 \mathrm{~V}$ over the $\mathrm{m} / \mathrm{z}$ range of 400 to 1100 . The low resolution precursor of $m / z(366.1 \pm 0.25)$ is shown in Figure $2 \mathrm{~d}$. The doubly protonated glycosylated conotoxin at $m / z 966.7$ and a slightly more intense monosodium adduct are observed. This increased tendency of metal cationized glycopeptides to lose the glycan moiety has been reported before [5]. In addition, several ion signals in the low and mid $\mathrm{m} / \mathrm{z}$ range are present in the precursor ion experiment and have the same or $60 \%$ more abundance than the glycopeptide. This result is contrasted by the high resolution precursors of $\mathrm{m} / \mathrm{z}$ (366.14 \pm 0.02) experiment (see Figure 2e). A clear increase in selectivity is achieved. The glycopeptidederived ion signals form the most intense ion signals in the precursor ion spectrum, whereas the non-specific ion signals do not exceed $50 \%$ relative intensity. This means that a threefold increase in specificity is obtained when using high resolution, high accuracy fragment ion selection for this type of precursor ion experiments.

\section{Detection Limit}

In order to examine the sensitivity of the precursors of glycan fragments ions method, a dilution series of the synthetic glycopeptide GFYFNKPS*GYGSSSRRA (S* denotes the glycosylation site, two mannose residues attached) was prepared and precursors of $m / z$ (163.06) experiments were preformed for each dilution. Since a new nanoelectrospray needle was used for each experiment carry-overs were avoided. Figure 3 shows the precursor ion scan for a $17 \mathrm{nM}$ solution $(17 \mathrm{fmol} / \mu \mathrm{l}$; determined by amino acid analysis): An ion signal for the quadruply charged precursor at $m / z 551$ is observable with a signal to noise ratio of 3 whereas the glycopeptide is not visible in the corresponding normal mode mass spectrum (data not shown). Although lower detection limits have been described for unmodified peptides this method is one of the most sensitive methods described as yet for the analysis of glycopeptides. This fairly high detection limit results from the 


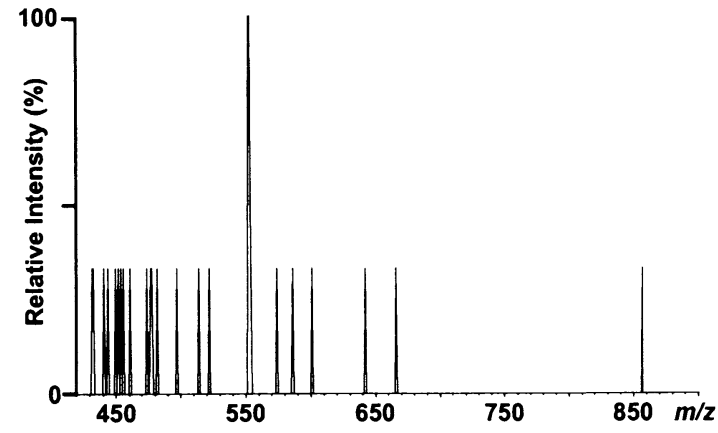

Figure 3. A high resolution precursor of $m / z 163$ scan showing the detection limits of the glycan derived fragment ion scan. The sample analyzed was a synthetic glycopeptide at a concentration of $17 \mathrm{nM}$ or $17 \mathrm{fmol} / \mu \mathrm{l}$.

low ionization efficiency of glycosylated peptides as compared to unmodified peptide species.

\section{Applications of the Precursor Ion Scanning Method}

Two isoforms of a secreted recombinant urokinase plasminogen activator receptor (UPAR) were separated by SDS-PAGE. These isoforms had been expressed in different cell lines and it was unclear whether the differences in electrophoretic mobility resulted from a truncation of the proteins or from differential modifications. As UPAR has four putative N-glycosylation sites, three of which were previously characterized as being glycosylated, it seemed feasible that the different mobilities of these two proteins were caused by a different glycosylation pattern due to the expression in different cell lines. The two gel bands (indicated as F2 and F3 hereafter) were excised and in-gel digested prior to desalting and concentration on microcolumns as described above.

Figures $4 \mathrm{a}$ and $5 \mathrm{a}$ depict the mass spectra of the peptide mixtures obtained after tryptic digestion of F2 and F3, respectively. Clear differences are observable in the mass spectra. On closer examination of the spectra,

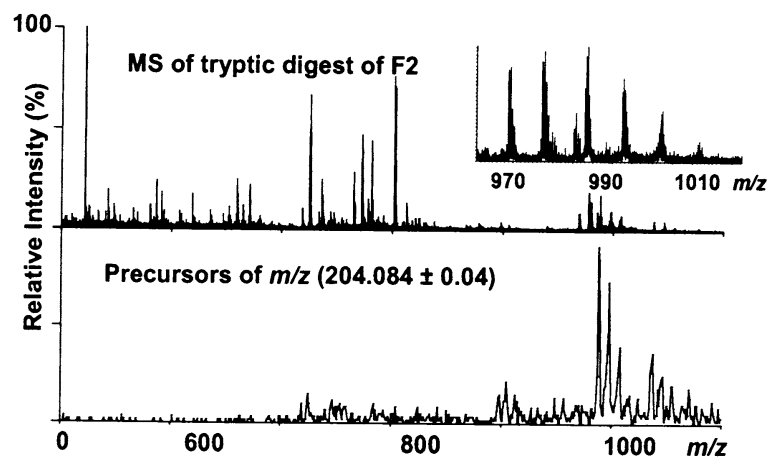

Figure 4. (a) displays the mass spectrum of a UPAR F2 tryptic digest. The inset shows the $\mathrm{m} / \mathrm{z}$ range of the glycopeptide. (b) shows the high resolution precursors of 204 scan of this UPAR F2 digest at the optimized collision energy settings. An intense signal is observed at $\mathrm{m} / \mathrm{z} 988$ for this isoform of UPAR.

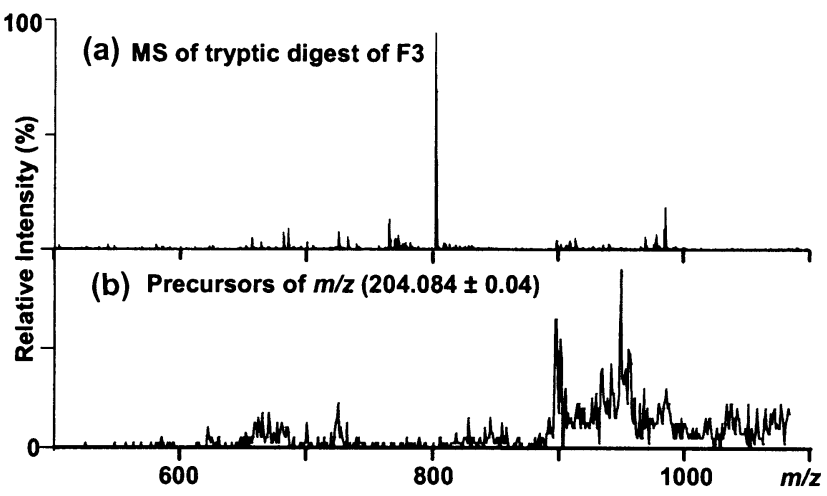

Figure 5. (a) is the mass spectrum of the F3 UPAR tryptic digest and (b) is the high resolution precursors of $\mathrm{m} / \mathrm{z} 204$ scan of this F3 digest. This F3 isoform displays its most intense signal at $\mathrm{m} / \mathrm{z}$ of 898.

some of the differences are only caused by the intensities of the ion signals. However, it is time-consuming to scan through the two spectra in an effort to ascertain differences. Thus, in order to determine if the differences between these two recombinant proteins were caused by different glycosylation patterns we performed a precursors of $\mathrm{m} / \mathrm{z}(204.08 \pm 0.02)$ experiment on the two samples and compared these scans. The collision energy was ramped during precursor ion scan from 25 to $30 \mathrm{~V}$ in the $\mathrm{m} / \mathrm{z}$ range of 400 to 1200 in order to induce the formation of the HexNAc oxonium ions while minimizing peptide backbone cleavages. Figures $4 \mathrm{~b}$ and $5 \mathrm{~b}$ show the two high resolution, high accuracy precursor ion scans. The two precursor ion spectra show clear differences in the glycosylation pattern for the F2 and F3 scans. The F2 sample shows the high intensity ion signals for one major ion signal at $\mathrm{m} / \mathrm{z} 998$ accompanied by its sodium adducts (Figure $4 b$ ). Some minor ion signals around $\mathrm{m} / \mathrm{z} 900$ and 1050 are also observable. This glycosylation profile is contrasted by the one observed for F3 as shown in Figure 5b. Intense ion signals at $\mathrm{m} / \mathrm{z} 898$ and 950 are observable in addition to a rather high background between $\mathrm{m} / \mathrm{z} 900$ and 1100 , which indicates highly heterogeneous glycosylation.

The precursors giving rise to major ion signals in the precursor ion experiment were selected for product ion experiments. In order to obtain peptide sequences from the product ion experiment, i.e., to induce peptide backbone fragmentation which is a secondary process, product ion spectra were collected with collision energy settings higher than normally used for peptide fragmentation (see above).

The product ion spectrum of the triply charged precursor at $\mathrm{m} / \mathrm{z}$ 988.09, derived from sample F2, is shown in Figure 6. The major ion signals in the lower and mid $\mathrm{m} / \mathrm{z}$ range are attributed to glycan fragmentation and glycan-specific fragment ions respectively. Nevertheless, it is possible to derive the complete peptide sequence GNSTHGCSSEETFLIDCR based on singly and doubly charged y fragment ions (singly charged y-ions are marked with dotted lines). It was not 


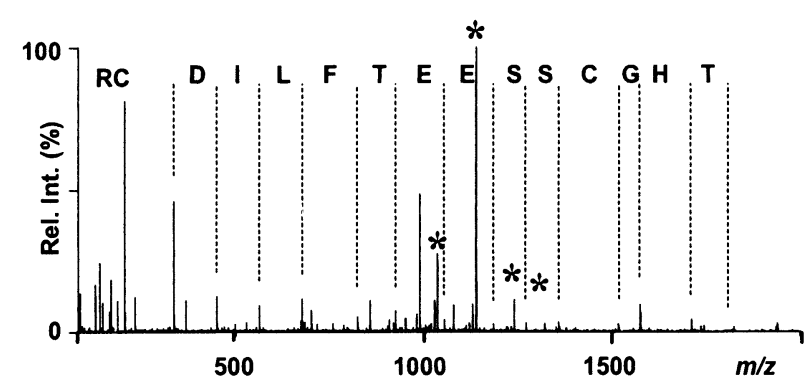

Figure 6. Product ion spectrum of the triply charged precursor at $\mathrm{m} / \mathrm{z} 988$, i.e., the predominant ion in the F2 the precursors of $\mathrm{m} / \mathrm{z}$ 204 scan spectrum. The sequence derived from the peptide fragmentation is shown above the spectrum. Singly charged y-ions are marked with dotted lines and asterisks demarcate ions giving rise to glycan sequence information.

possible to localize the glycosylation site based on the fragmentation pattern since the glycan was cleaved off as a primary fragmentation. However, since there is only one asparagine residue/NXT motif (marked in bold face) it is safe to assign the $\mathrm{N}$-glycosylation site, especially as previous studies have shown that the marked asparagine residue is a glycosylation site [17]. The glycan structure/sequence could be determined to be $(\mathrm{HexNAc})_{2}-(\mathrm{Hex})_{3}$ with the HexNAc residues attached to the peptide. Based on this previous study, it is presumed that this pentasaccharide adopts a biantennary, though more experiments are required to confirm both notions.

The product ion spectrum of the quadruply charged precursor at $\mathrm{m} / \mathrm{z} 898.13$, which gave rise to one of the major ion signals in the precursor ion experiment of the F3 sample, is shown in Figure 7. The lower $m / z$ range is dominated by the HexNAc and the HexHexNAc oxonium ion at $\mathrm{m} / \mathrm{z} 204$ and 366 in addition to prominent peptide-derived $\mathrm{a}$ and $\mathrm{b}$ ions which allow the identification of the glycosylated peptide. In contrast to this, the higher $\mathrm{m} / \mathrm{z}$ range is dominated by fragment ions corresponding to the fragmentation of the glycan. No

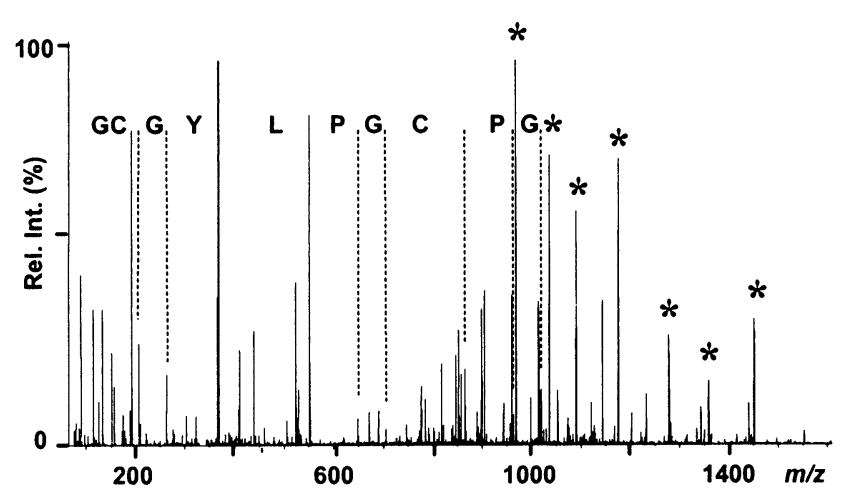

Figure 7. Product ion spectrum of the predominant ion in the F3 precursors of $\mathrm{m} / \mathrm{z} 204$ scan at $\mathrm{m} / \mathrm{z}$ of 898 ; here the ions that provide glycan structural information are in the higher $\mathrm{m} / \mathrm{z}$ range. The peptide sequence derived from the product ion spectrum is displayed above the spectrum. Singly charged b-ions are marked with dotted lines and asterisks demarcate ions giving rise to glycan sequence information. obvious peptide-derived $\mathrm{b}$ or y ions could be identified in this part of the product ion spectrum. Nevertheless, the product ion information was sufficient to identify the peptide sequence as GCGYLPGCPGSNGFHNNDTFHFLK. Even though it was not possible to unambiguously identify the site of glycosylation, the NXT motif (marked in bold face) strongly suggest $\mathrm{N}$-glycosylation on that asparagine residue; this notion is supported by previous studies [17]. The glycan structure/sequence seems to be identical to the glycan structure determined for the precursor described above.

Other ions giving rise to a signal in the precursor of 204.08 scans were also fragmented and found to be glycosylated peptides; a detailed study will be published elsewhere. These data shows the utility, specificity, and sensitivity of the high resolution precursor ion scan for the detection of glycopeptides.

\section{Conclusion}

In this study, glycopeptide-specific precursor ion scanning was re-examined to take advantage of the recently introduced high resolution, high accuracy fragment ion selection on quadrupole TOF type hybrid tandem mass spectrometers. The data were compared with low resolution experiments resembling those on triple quadrupole tandem mass spectrometers, the traditional instrument for the precursor ion experiment. The low resolution experiments show a clear lack of specificity, especially for the Hex and HexHexNAc oxonium ions at $\mathrm{m} / \mathrm{z} 163$ and 366, respectively. The use of high resolution, high accuracy fragment ion selection significantly reduced the number of nonspecific ion signals in the precursor ion spectrum. Specificity was also increased if the applied collision energies were optimized for maximum oxonium ion generation and minimum peptide backbone fragmentation and this turned out to be crucial for sensitive and selective precursor ion experiments. The good selectivity of the precursors of $\mathrm{m} / \mathrm{z} 204$ experiment, which specifically chooses HexNAc-forming precursors, was not further improved by using high resolution, high accuracy fragment ion selection under low collision energy conditions.

However, the fact that glycans often give rise to multiple "reporter" ions helps to increase the confidence in identifying glycopeptides in complex peptide mixtures even when the specificity of one precursor ion experiment is suboptimal due to isobaric interferences.

The detection limits for the precursors of $\mathrm{m} / \mathrm{z} 163$ were determined to be below $20 \mathrm{nM}$ and should be in the same range for the precursors of $m / z 204$ experiment and slightly poorer (i.e., higher) for the precursors of $\mathrm{m} / \mathrm{z} 366$ experiment since this oxonium ion is normally of lower abundance than the oxonium ions at $\mathrm{m} / \mathrm{z} 163$ and 204 (if multiple oxonium ions are present).

The improved precursor ion method with optimized collision energies and high resolution fragment ion selection was used to investigate the different glycosylation pattern of secreted urokinase plasminogen activa- 
tor receptor proteins, expressed in two different cell lines. Clear differences could be determined explaining the different migration behaviors under SDS-PAGE conditions. These improved high resolution precursor ion scanning experiments can therefore be used to identify and localize unknown glycosylated peptides in peptide digest mixtures. The increased specificity results in less time being spent on acquiring MS/MS data from peptides that produce fragments of the same nominal mass.

\section{Acknowledgments}

The authors thank Professor Matthias Mann (Center for Experimental BioInformatics, Department of Biochemistry and Molecular Biology, University of Southern Denmark, Odense, Denmark) for providing instrument time on the QSTAR mass spectrometer. The authors acknowledge Dr. B. Hambe (Department of Clinical Chemistry, Lund University, University Hospital, Malmö, Sweden) and Professors J. Stenflo (Marine Biological Laboratory, Woods Hole, MA, USA and Department of Clinical Chemistry, Lund University, University Hospital, Malmö, Sweden), B. Furie and B. C. Furie (Center for Hemostasis and Thrombosis Research, Harvard Medical School and Beth Israel Deaconess Medical Center, Boston, MA, USA, and Marine Biological Laboratory, Woods Hole, MA, USA) providing the purified glycosylated Conus textile conotoxin. They also acknowledge Dr. Gunilla Høyer-Hansen (Copenhagen University Hospital) for providing the UPAR F2 and F3 samples. This study is part of the activities of the Center for Experimental Bioinformatics sponsored by the Danish National Research Foundation and the Danish Biotechnology Instrument Center.

\section{References}

1. Apweiler, R.; Hermjakob, H.; Sharon, N. On the Frequency of Protein Glycosylation, as Deduced from Analysis of the SWISS-PROT Database. Biochim. Biophys. Acta 1999, 1473, 4-8.

2. Hart, G. W.; Greis, K. D.; Dong, L. Y.; Blomberg, M. A.; Chou, T. Y.; Jiang, M. S.; Roquemore, E. P.; Snow, D. M.; Kreppel, L. K.; Cole, R. N. O-Linked N-Acetylglucosamine: The "YinYang" of Ser/Thr Phosphorylation? Nuclear and Cytoplasmic Glycosylation. Adv. Exp. Med. Biol. 1995, 376, 115-123.

3. Carr, S.; Huddleston, M.; Bean, M. Selective Identification and Differentiation of N- and O-Linked Oligosaccharides in Glycoproteins by Liquid Chromatography-Mass Spectrometry. Protein Sci. 1993, 2, 183-196.

4. Ritchie, M. A.; Gill, A. C.; Deery, M. J.; Lilley, K. Precursor Ion Scanning for Detection and Structural Characterization of Heterogeneous Glycopeptide Mixtures. J. Am. Soc. Mass Spectrom. 2002, 13, 1065-1077.

5. Huddleston, M. J.; Bean, M. F.; Carr, S. A. Collisional Fragmentation of Glycopeptides by Electrospray Ionization
LC/MS and LC/MS/MS: Methods for Selective Detection of Glycopeptides in Protein Digests. Anal. Chem. 1993, 65, 877884 .

6. Steen, H.; Kuster, B.; Fernandez, M.; Pandey, A.; Mann, M. Detection of Tyrosine Phosphorylated Peptides by Precursor Ion Scanning Quadrupole TOF Mass Spectrometry in Positive Ion Mode. Anal. Chem. 2001, 73, 1440-1448.

7. Steen, H.; Kuster, B.; Fernandez, M.; Pandey, A.; Mann, M. Tyrosine Phosphorylation Mapping of the Epidermal Growth Factor Receptor Signaling Pathway. J. Biol. Chem. 2002, 277, 1031-1039.

8. Petersson, A.-S.; Steen, H.; Kalume, D. E.; Caidahl, K.; Roepstorff, P. Investigation of Tyrosine Nitration in Proteins by Mass Spectrometry. J. Mass Spectrom. 2001, 36, 616-625.

9. Steen, H.; Mann, M. Analysis of Bromotryptophan and Hydroxyproline Modifications by High-Resolution, High-Accuracy Precursor Ion Scanning Utilizing Fragment Ions with Mass-Deficient Mass Tags. Anal. Chem. 2002, 74, 6230-6236.

10. Shevchenko, A.; Wilm, M.; Vorm, O.; Mann, M. Mass Spectrometric Sequencing of Proteins from Silver-Stained Polyacrylamide Gels. Anal. Chem. 1996, 68, 850-858.

11. Gobom, J.; Nordhoff, E.; Mirgorodskaya, E.; Ekman, R.; Roepstorff, P. Sample Purification and Preparation Technique Based on Nano-Scale Reversed-Phase Columns for the Sensitive Analysis of Complex Peptide Mixtures by Matrix-Assisted Laser Desorption/Ionization Mass Spectrometry. J. Mass Spectrom. 1999, 34, 105-116.

12. Wilm, M.; Mann, M. Analytical Properties of the Nanoelectrospray Ion Source. Anal. Chem. 1996, 68, 1-8.

13. Medzihradszky, K. F.; Gillece-Castro, B. L.; Settineri, C. A.; Townsend, R. R.; Masiarz, F. R.; Burlingame, A. L. Structure Determination of O-Linked Glycopeptides by Tandem Mass Spectrometry. Biomed. Environ. Mass Spectrom. 1990, 19, 777781.

14. Steen, H.; Petersen, J.; Mann, M.; Jensen, O. N. Mass Spectrometric Analysis of a UV Cross-Linked Protein-DNA Complex: Tryptophans 54 and 88 of E. coli SSB Cross-Linked to DNA. Protein Sci. 2001, 10, 1989-2001.

15. Rigby, A. C.; Lucas-Meunier, E.; Kalume, D. E.; Czerwiec, E.; Hambe, B.; Dahlqvist, I.; Fossier, P.; Baux, G.; Roepstorff, P.; Baleja, J. D.; Furie, B. C.; Furie, B.; Stenflo, J. A Conotoxin from Conus textile with Unusual Posttranslational Modifications Reduces Presynaptic Ca2+ Influx. Proc. Natl. Acad. Sci. U.S.A. 1999, 96, 5758-5763.

16. Kalume, D. E.; Stenflo, J.; Czerwiec, E.; Hambe, B.; Furie, B. C.; Furie, B.; Roepstorff, P. Structure Determination of Two Conotoxins fromConus textile by a Combination of Matrix-Assisted Laser Desorption/Ionization Time-of- Flight and Electrospray Ionization Mass Spectrometry and Biochemical Methods. J. Mass Spectrom. 2000, 35, 145-156.

17. Ploug, M.; Rahbek-Nielsen, H.; Nielsen, P. F.; Roepstorff, P.; Dano, K. Glycosylation Profile of a Recombinant UrokinaseType Plasminogen Activator Receptor Expressed in Chinese Hamster Ovary Cells. J. Biol. Chem. 1998, 273, 13933-13943. 